\title{
Cytology material is equivalent to tumor tissue in determining mutations of BRCA $1 / 2$ genes in patients with tubo-ovarian high grade serous carcinoma
}

Andreja Gornjec ${ }^{1}$, Srdjan Novakovic ${ }^{2}$, Vida Stegel ${ }^{2}$, Marko Hocevar $^{3}$, Ziva Pohar Marinsek ${ }^{4}$, Barbara Gazic ${ }^{5}$, Mateja Krajc ${ }^{6}$ and Erik Skof ${ }^{7 *}$

\begin{abstract}
Background: High-grade serous ovarian cancer is a detrimental disease. Treatment options in patients with a recurrent disease are dependent on BRCA1/2 mutation status since only patients with known BRCA mutation are eligible for treatment with poly(ADP-ribose) polymerase inhibitors (PARPi). The aim of this study was to compare concordance of BRCA mutation analyses from cytological samples (CS) with BRCA mutation analyses from histological formalin fixed paraffin embedded (FFPE) samples.
\end{abstract}

Methods: Mutation analysis of BRCA1 and BRCA2 genes was performed in 44 women diagnosed with primary or recurrent high-grade ovarian cancer from three different samples: blood, cytological sample (ascites, pleural effusion and enlarged lymph nodes) and tumor tissue. Results from all three samples were compared.

Results: Among 44 patients, there were 15 germline mutations and two somatic mutations. A 100\% concordance was found between cytological and histologic samples.

Conclusion: There is a 100\% concordance in BRCA mutation testing between cytological and histologic samples. BRCA mutation testing from CS could replace testing from FFPE tissue in clinical decision making in ovarian cancer patients.

Trial registration: The study was retrospectively registered at ISRCTN registry on 24/11/2015 - ISRCTN42408038.

Keywords: High-grade serous cancer, BRCA1/2 mutation, BRCA1/2 mutation testing, Cytological samples, Formalin fixed paraffin embedded samples

\section{Background}

Ovarian cancer $(\mathrm{OC})$ is in $3,5-18 \%$ associated with germline mutations in BRCA1 and BRCA2 genes [1-4]. Germline BRCA1 and BRCA2 mutations are most common in high grade serous histologic type (high grade serous cancer - HGSC), the most frequent type of epithelial ovarian cancer, and appear in 16-39.2\% of cases [5-10]. In addition, somatic $B R C A 1 / 2$ mutations are present in $3.0-7.9 \%$ of cases in ovarian cancer patients $[8,11,12]$. Germline BRCA mutations have a positive impact on overall survival and platinum responsiveness of ovarian

\footnotetext{
* Correspondence: eskof@onko-i.si

${ }^{7}$ Department of Medical oncology, Institute of Oncology Ljubljana, Zaloška cesta 2, 1000 Ljubljana, Slovenia

Full list of author information is available at the end of the article
}

cancer patients [13]. Somatic BRCA mutations have the same impact $[9,14,15]$. Furthermore, another important hallmark of BRCA1/2-associated ovarian cancers is sensitivity to poly(ADP-ribose) polymerase inhibitors (PARPi) [9].

In Europe, an oral potent PARP inhibitor Olaparib has been added to treatment guidelines for patients with platinum sensitive recurrent serous ovarian, tubal and primary peritoneal cancers with known $B R C A$ mutation. Irrespective of whether the origin of the $B R C A$ mutation is germline or somatic, PARP inhibitor Olaparib significantly prolongs progression-free survival (PFS) versus placebo in patients with platinum-sensitive recurrent serous ovarian cancer $[9,12,14]$. Before the enrolment of treatment with PARP inhibitor $B R C A$ mutation has to 
be proven (germline or somatic). BRCA status is not known in majority of the ovarian cancer patients. Therefor genetic testing must precede enrolment of the treatment.

Germline BRCA mutation testing is at this time done only after the genetic counseling in patients with positive familial history for inherited breast and ovarian cancer syndrome and in some patients with known breast or ovarian cancer. Aim of this genetic testing is primary and secondary preventive. Germline $B R C A$ mutations are analyzed from peripheral blood cells. Somatic $B R C A$ mutation tests have to be done from tumor cells.

Due to the intratumoral genetic heterogeneity (ITH) in primary and recurrent cancers, [16-18] intratumoral widespread regional diversities observed in mutational, copy number and gene expression landscapes using derived samples of primary tumor and metastatic sites [19] and importance of presence of somatic mutations, $[15,18,20-23]$ the best strategy for BRCA mutation testing would be from tumor cells. Tumor cells for $B R C A$ testing can be obtained from histologic samples gained during operative procedures or from cytological material derived from minimally invasive procedures. Genetic testing from histologic samples is usually performed from formalin-fixed paraffin-embedded (FFPE) tissue samples, where quality of isolated DNA could be a problem. Genetic testing would be ideally done from fresh or fresh frozen tissue samples. In most of the cases it is not feasible, because of spatial and logistic problems and time divergence of genetic testing and gaining of tissue samples, which are most of the times done after primary or interval cytoreduction [24-26]. On the other hand genetic material extracted from cytological samples is of good quality and relatively easy accessible, which enables genetic testing. Ovarian cancer is in $75 \%$ of patients diagnosed at advanced stages where tumor has already spread to peritoneal surfaces and cells are present in peritoneal free fluid. Recurrent disease often presents with malignant ascites and peritoneal and abdominal metastases and is seldom treated with operative procedure where tissue samples could be taken. Cytological material is easily accessible before nearly every treatment enrolment without invasive procedures such as surgical operation. To our knowledge, only two studies did genetic analysis on DNA isolated from ascites derived cancer cells and one on DNA isolated from cells in pleural effusions of patients with HGSC. Castellarin et all performed whole exome sequencing on tumor cells harvested from ascites. They provided the first evidence at single nucleotide resolution that recurrent high grade serous cancer arises from multiple clones present in the primary tumor with negligible accumulation of new mutations during standard treatment [16]. Results from Choi et all confirmed the theory that ascites cells are shed from solid tumor lesions and showed that ascites derived cancer cells integrated ITH and found that mutation profiling results of ascites cells in the ovarian HGSC patients represent and integrate entire mutational landscape of a given HGSC and cover the majority of somatic mutations found across regional biopsies of the corresponding tumors [22]. The study from Shah et all compared genomic alterations results from DNA extracted from pleural effusion, FFPE and matched blood from 4 HGSC patients. They confirmed that cytological material could represent good material for genomic alterations identification with next generation sequencing and demonstrated that very little DNA $(<50 \mathrm{ng})$ can yield extremely high coverage sequence data [18]. Considering all characteristics of HGSC as disease with lots of recurrences, eventual chemoresistance, somatic reverting mutations and also ITH with limited availability of FFPE before every treatment enrolment, knowing that targeted personalized therapy is future directive for HGSC treatment and with confidence data that ascites tumor cells represent entire mutational profiling landscape of given HGSC, cytology could be equivalent to FFPE and even represent the most accurate mutation profiling result for a given HGSC in a certain time of disease progression.

The aim of our study was to compare $B R C A$ mutation profiling of cytological material (cells in ascites, cells from pleural effusion or cells derived from fine needle aspiration of lymph node metastasis) with histologic samples from tumor tissue obtained during operation.

We expected to confirm $100 \%$ concordance between $B R C A$ mutation testing from cytological samples (CS) in comparison to FFPE. We wanted to determine if $B R C A 1 / 2$ testing from CS can be used instead of FFPE in HGSC patients.

\section{Methods}

From October 2015 to December 2017 patients with primary or recurrent ovarian, fallopian tube and primary peritoneal high-grade serous cancer (subsequently collectively referred to as high-grade serous cancer - HGSC) who were treated at the Institute of Oncology Ljubljana, were included in the study.

The study was approved by National Ethics Committee and Institutional Ethics Committee of Institute of Oncology Ljubljana (Ljubljana, Slovenia), 27/07/2015, NMEC 100/05/ 15. All samples from the patients in this study were obtained after appropriate informed written consents. The study was retrospectively registered at ISRCTN registry on 24/11/2015 (ISRCTN42408038).

Inclusion criteria were as follows: age $<70$ Years, WHO performance status $0-1$, histologically verified high grade serous ovarian, tubal or primary peritoneal cancer, presence of ascites or enlarged peripheral lymph nodes, availability of FFPE tissue samples derived at surgical operation, 
indication for enrolment of treatment with platinum agents, normal kidney function (creatinine $<100 \mu \mathrm{mol} / \mathrm{l}$, endogenous creatinine clearance $(\mathrm{ECC})>75 \mathrm{ml} / \mathrm{l}$ ).

Exclusion criteria were as follows: types other than HGSC of ovarian cancer, unavailability of FFPE tissue samples derived at surgical operation, no malignant cells in ascites or enlarged peripheral lymph nodes, WHO performance status $\geq 2$, history of presence of other cancer (except squamous cell skin cancer, uterine cervical cancer, breast cancer and in situ breast cancer).

Only patients with available three sample types (CS, blood and FFPE) were considered eligible.

All included patients performed genetic counselling and testing for germline BRCA1/2 mutation at Institute of Oncology Ljubljana.

\section{Cytological samples evaluation}

Cytological samples were obtained either at ultrasonography guided transabdominal puncture, with thoracic paracentesis or with fine needle biopsy in ambulatory setting under local anesthesia. Samples (at least $30 \mathrm{ml}$ of ascites or pleural effusion) were promptly sent to the Cytology department. All included patients (including patients with primary inoperable disease who were treated with neoadjuvant chemotherapy) had confirmed disease (HGSC) by FFPE - described in inclusion/exclusion criteria. Ascites and pleural effusion cells were pelleted by classical centrifugation $(2700 / 10 \mathrm{~min})$. Two smears were prepared from each sample according to our standard protocol and stained with Giemsa or Papanicolau. To a part of each sample a cell medium $(20 \%$ bovine serum albumin, 5\% EDTA, $4 \mathrm{~N} \mathrm{NaOH}$ in phosphate buffer solution with 1000.000 IE of penicillin and $80 \mathrm{mg}$ Garamycin), routinely prepared in our laboratory, was added. Cell medium was also added to fine needle punctate of enlarged lymph nodes. Sample was cytocentrifugated and prepared for cythopathological review. One cythopathologist reviewed all prepared samples and assessed the presence of adenocarcinoma tumor cells in the sample. Samples were then sent to Molecular department for $B R C A$ testing.

\section{Histological samples evaluation}

Histological samples were obtained at interval cytoreductive surgery or at primary surgical operation and sent to the Pathology department. All samples were formalin fixed and paraffin embedded. Hematoxylin and Eosin (HE) stained slides were prepared from each paraffin block. One pathologist reviewed all HE-slides of each patient, confirmed the tumor type, evaluated the percentage of tumor cells on each slide and chose a representative paraffin block where at least $60 \%$ of tumor tissue was present. From the selected block samples were prepared for molecular analysis and sent to the Department of Molecular Diagnostics for BRCA testing.

\section{Extraction of DNA}

In the study the DNA samples extracted from whole blood, ascites and formalin-fixed paraffin embedded tumor tissue were used.

From the whole blood and ascites, the DNA was extracted using InnuPREP Master Blood kit (Analytik Jena, Thuringia, D). From FFPE tumor tissue, the DNA was extracted using GeneRead DNA FFPE Kit (QiagenGmbH, Hilden, Germany)) from manually macro-dissected areas annotated by a pathologist by scraping directly off unstained standard glass slides $(10 \mu \mathrm{m})$. Hematoxylin-eosin staining of the first sectioned slide was performed to visualize the presence of tumor cells, and to guide macro-dissection on unstained duplicate slides and to determine the area of the tissue cores. After the isolation DNA concentration was spectrophotometrically measured at $280 / 260 \mathrm{~nm}$.

\section{Next generation sequencing - NGS}

The coding sequence and exon/intron boundaries on DNA isolated from blood and ascites were enriched using Nextera DNA Library Preparation Kit in combination with TruSight ${ }^{\circ}$ Cancer Panel (Illumina, San Diego, USA). The coding sequence and exon/intron boundaries of BRCA1 and BRCA2 genes on DNA isolated from FFPE tumor tissue and ascites were amplified using TruSeq Custom Amplicon Kit in combination with primer pools in Targeted DNA Repair_2 Gene consortium panel kit - TSCA-BRCA (Illumina, San Diego, USA). Before the library preparation the DNA quality and quantity was assessed using Infinium FFPE QC and DNA Restoration Kit (Illumina). Next generation sequencing was performed on Illumina MiSeqDx Sequencing System (Illumina) according to manufacturer's protocol. Secondary data analysis and base calling was performed by MiSeq Reporter Software 2.5.1, using DS Somatic as variant caller (Illumina). VCF v4.1 files generated during secondary analysis of sequencing data were imported into Illumina Variant Studio software for variant annotation and filtering. When TruSight ${ }^{\circ}$ Cancer Panel was used, more than 95\% of targeted regions in BRCA1 and BRCA2 genes were covered $>150 x$. Estimated limit of detection for this method was $10 \%$ of mutant allele in the background of wild type for single nucleotide variants (SNV), and 25\% for insertion and deletions (indel) (up to $11 \mathrm{bp}$ ). When TSCA-BRCA was used, more than $95 \%$ of targeted regions in $B R C A 1$ and $B R C A 2$ genes were covered $>500 \mathrm{x}$. Estimated limit of detection for this method was $5 \%$ for SNV and $10 \%$ for indel (up to $11 \mathrm{bp}$ ). 
Classification of detected variants was done through Illumina Variant Interpreter Software (Illumina).

In silico mutation prediction analysis was performed applying online bioinformatics tool Alamut ${ }^{\circ}$ Visual 2.11.

\section{Direct sequencing}

Direct Sanger sequencing was performed to validate mutations detected by NGS. For direct DNA sequencing, the samples were bidirectionally sequenced on an automated ABI 3500 genetic analyzer (Applied Biosystems, Foster City, CA). Estimated limit of detection is $20 \%$ of mutant allele in the background of wild type for SNV and indels (up to $11 \mathrm{bp}$ ).

\section{MLPA analysis}

For detection of large deletions and insertions the MRC Holland MLPA (Multiplex Ligation - dependent Probe Amplification) kit P045-B1 for BRCA2, and P002 for $B R C A 1$ was used according to manufacturer's instructions (MRC Holland, Amsterdam, Nederland).

Nucleotide variants were classified according to ACMG guidelines [27, 28].

\section{Statistical analysis}

The study was designed to have $80 \%$ power of research, to prove $\geq 90 \%$ certainty of cytology, knowing that cytology specificity is at least $99 \%$, where risk for $\alpha$ error is $5 \%$ or less. To meet these criteria, we had to include at least 40 patients [29].

Descriptive statistics was used to describe basic features of the data. For differences of BRCA mutation test results between CS, FFPE and blood group $\mathrm{Chi}^{2}$ test was used. $P$ value $<0,05$ was considered significant.

Statistical analysis was done with IBM SPSS 25 software.

\section{Results}

Overall 44 patients with HGSC and results of BRCA testing from three sample types (CS, blood and FFPE) were included in this study. Their clinical characteristics are presented in Table 1. Mean age at the time of diagnosis was 59.8 years (range 40-77). Eight out of 44 patients (18.1\%) had a positive family history for hereditary breast and ovarian cancer and 6/44 (13.6\%) had a known diagnosis of breast cancer before HGSC was diagnosed. Four out of these six patients $(66.7 \%)$ had also a positive familial history. Mean time from breast cancer diagnosis to ovarian cancer diagnosis was 21,33 years (range 9-28). There was not a single breast cancer relapse in these patients and so free interval from breast cancer to ovarian cancer was more than 20 years. All clinical characteristics of disease including serum tumor markers at the time of sample collection were in accordance with ovarian cancer.
Table 1 Clinical characteristics of 44 patients with HGSC

\begin{tabular}{|c|c|}
\hline Age at diagnosis & years \\
\hline Mean & 59,8 \\
\hline Range & $40-77$ \\
\hline Localisation of HGSC at diagnosis & n (\%) \\
\hline Ovarian cancer & $34(77,3)$ \\
\hline Fallopian tube cancer & $6(13,6)$ \\
\hline Primary peritoneal serous cancer & $4(9,1)$ \\
\hline FIGO Stage & n (\%) \\
\hline IA & $0(0)$ \\
\hline IB & $1(2,3)$ \\
\hline IC & $2(4,5)$ \\
\hline$\|$ & $0(0)$ \\
\hline$\| I \mid A 1$ & $1(2,3)$ \\
\hline$\| I I C$ & $30(68,2)$ \\
\hline IV & $10(22,7)$ \\
\hline Family history & n (\%) \\
\hline Positive & $8(18,2)$ \\
\hline Negative & $34(77,3)$ \\
\hline Unknown & $2(4,5)$ \\
\hline Breast cancer & n (\%) \\
\hline Yes & $6(13,6)$ \\
\hline No & $26(59,1)$ \\
\hline Unknown & $12(27,3)$ \\
\hline
\end{tabular}

Legend: HGSC high grade serous cancer, FIGO International Federation of Gynecology and Obstetrics

\section{$B R C A$ mutation testing results} Blood BRCA1/2 testing results

Fifteen $B R C A 1 / 2$ mutations $(15 / 44,34.1 \%)$ were identified in peripheral venous blood. Fourteen patients had a $B R C A 1$ mutation and one had a BRCA2 mutation. All other patients had wild type $B R C A$ genes. All mutations were known pathogenic mutations. The most common BRCA1 mutation was c.843_846delCTCA p.(Ser282Tyrfs*15) which was present in three patients. BRCA1 mutations c.844_850dupTCATTAC p.(Gln284Leufs*5), deletion of exons 4-9 and c. $1687 \mathrm{C}>\mathrm{T}$ p. $\left(\mathrm{Gln} 563^{*}\right)$ were all present in two patients. All other mutations were present in only one patient (Table 2).

\section{Cytological BRCA1/2 testing results}

Cytological samples for DNA analysis were derived from ascites fluid (AS) in 34, enlarged lymph nodes (ELN) in 6 and pleural effusion (PE) in four patients (Table 1). In 20 patients cytological samples were taken before primary treatment of newly diagnosed disease and in 24 patients before enrolment of treatment of recurrent disease. Percentage of tumor cells present per sample was on average $53.1 \%$ (range 1-95\%). Seventeen BRCA1/2 mutations were 


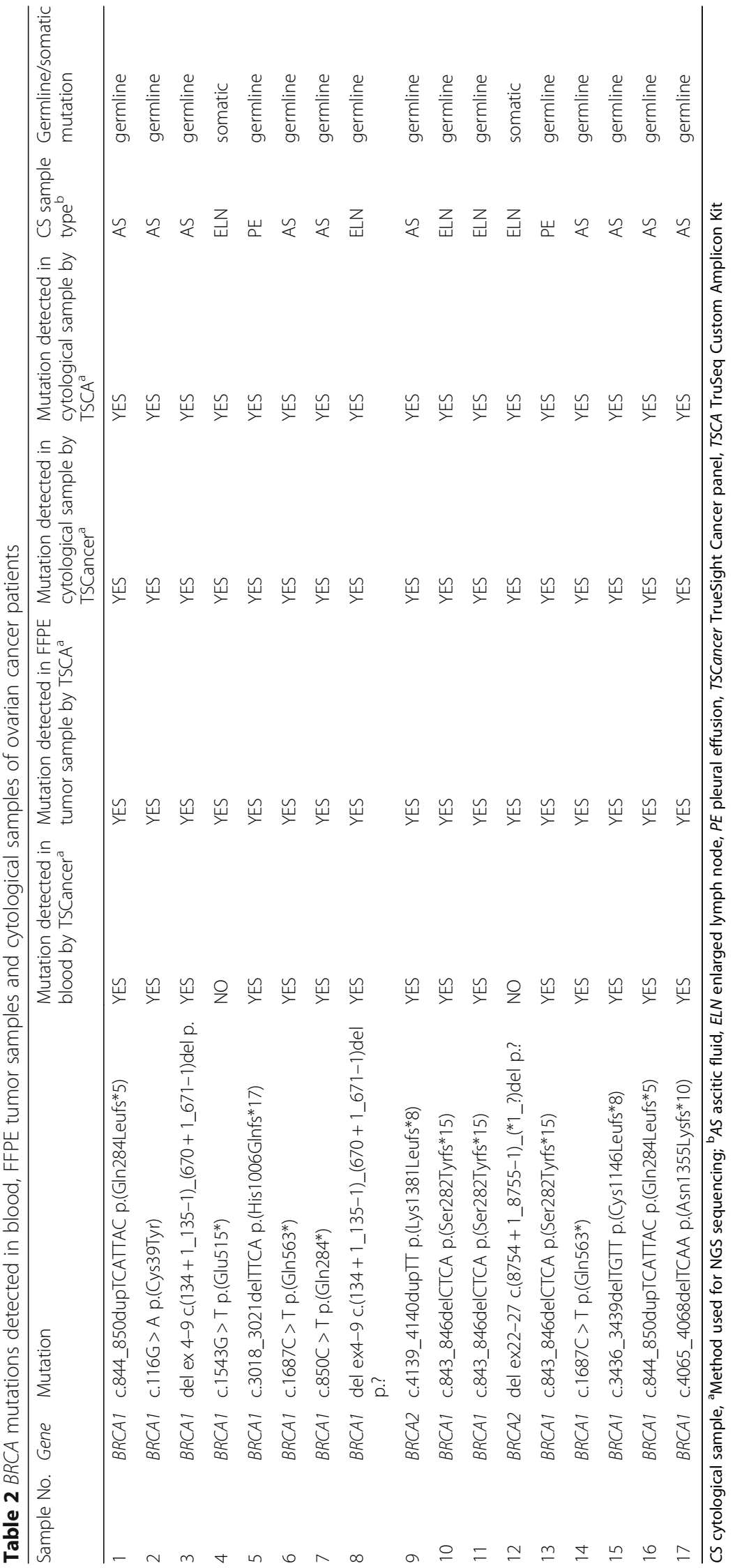


identified in cytological samples: $15 B R C A 1$ and $2 B R C A 2$. $15 B R C A 1 / 2$ mutations matched 15 germline BRCA1/2 mutations identified in blood samples. Two additional $B R C A 1 / 2$ mutations, which were not identified in blood samples, were considered as somatic: one $B R C A 1$ and one BRCA2 (Table 3). Both somatic mutations BRCA1 c.1543G > T p.(Glu515*) and BRCA2 (del ex 22-27) are known pathogenic mutations. Both patients with somatic mutations had a negative family history and did not have a breast cancer.

\section{Histological BRCA1/2 testing}

In 19 patients samples for DNA analysis were obtained at interval cytoreductive surgery (after 4-6 cycles of chemotherapy) in 22 patients during primary surgeries and in 3 during surgical treatment of recurrent disease. Percentage of tumor cells present on FFPE block slide was on average $83.2 \%$ (range 65-95). Exactly the same 17 BRCA1/2 mutations which were identified in cytological samples were identified in histological samples (100\% concordance).

No additional somatic mutations were detected in tumors from patients with germline $B R C A$ mutations in cytological samples. All patients were previously tested for germline mutations from DNA extracted from the peripheral blood samples (Table 3 ).

\section{Discussion}

We postulated that BRCA1/2 mutation analyses from cancer cells derived from cytological samples like ascites, pleural effusion and enlarged lymph nodes would be concordant with $B R C A 1 / 2$ mutation analyses from FFPE tumor tissue. There is no doubt that tumor tissue (FFPE) is standard material for initial diagnosis of HGSC. However, in our study the $100 \%$ concordance in detection of germline $B R C A 1 / 2$ mutations between all three sample types (CS, FFPE and blood) was confirmed. Moreover, the $100 \%$ concordance was also observed between mutation detection in CS and FFPE in two cases of somatic mutations alluding that cytology samples could replace tumor tissue in determining $B R C A 1 / 2$ mutation status in patients with HGSC where FFPE is hardly available. This is true when cytological and FFPE tumor samples have expected allele frequency of mutations above frequencies defined as limits of detection of the method used for genotyping (e.g. if tumor cells are heterozygous for mutations, the percentage of tumor cells in the sample should be twice of the limit of detection).

Our data strongly support and upgrade studies from Shah at all, where pleural effusion from 4 HGSC patients was used as cytological sample for DNA extraction and characterization of genomic alterations [18] and Choi et all, where conservation of cancer related genes in ascites cells from 4 ovarian cancer patients was evaluated.
Since ovarian cancers are characterized by complex and changing genetic profile, $B R C A 1 / 2$ tumor testing results can potentially vary depending on disease stage and sample site [20]. There is also the possibility that inactivated (mutated) $B R C A 1 / 2$ gene can revert to a functional gene during disease treatment and progression, due to somatic reverting mutations (resulting in resistance to therapy to PARP inhibitors). However in our cohort no reverting somatic mutations in $B R C A$ genes were detected. As nearly half of patients included in our study did not have recurrence of the disease, this could induce bias, because reverting mutations could not have time to develop yet. Since the number of patients included in the study was too low to optimally address this issue, the reverting mutation analysis was not the aim of this study. Continuation of this study is already taking place to eliminate this bias.

Together with before mentioned data and possibility for mutation reversal during cancer progression and treatments, ascites cells are proven to be a very good resource for identifying genomic alterations [17, 30]. When using cytologicaly derived cancer cells DNA to detect $B R C A 1 / 2$ mutation, both germline and somatic $B R C A 1 / 2$ mutations are identified. Results are independent on tumor sampling site. This is an important advantage, as potentially only one test needs to be performed to identify all patients with deleterious $B R C A 1 / 2$ variants that may benefit from new molecular targeted treatment. To distinguish between germline and somatic BRCA mutations, additional blood test must be performed.

Incidence of BRCA mutations in our study was 34\%, which is among the highest reported. An estimated 16$39.2 \%$ of OC patients are likely to have either germline or somatic BRCA1/2 mutation according to the literature [6]. The highest reported incidence was from the study of Petrillo et all, were incidence of $B R C A$ mutations in HGSC patients was $39.2 \%$. Unfortunately, $B R C A$ mutations in their study were not specified to germline or somatic, so exact comparison with our study is not possible [10]. Our results confirmed results of Cvelbar et all who analyzed the incidence of germline BRCA mutations in ovarian cancer patients younger than 50 years in Slovenia and found 33.3\% incidence of $B R C A$ mutations [31]. Our study presents first data about the incidence of somatic mutations among HGSC patients in Slovenia. The low incidence of $4.5 \%$ of somatic mutations is in agreement with the results of other international studies. Study of Integrated Genomic Analyses of Ovarian Carcinoma reported 3\% incidence of somatic mutations and Geisler et all reported 6,8\% incidence of somatic mutations, though in their study where not only HGSCs, but all epithelial ovarian cancers assessed for $B R C A$ germline and somatic mutations [8, 32].

Since $34 \%$ of our patients had a germline BRCA1/2 mutation, it is surprising that only $8 / 44$ of them had a 


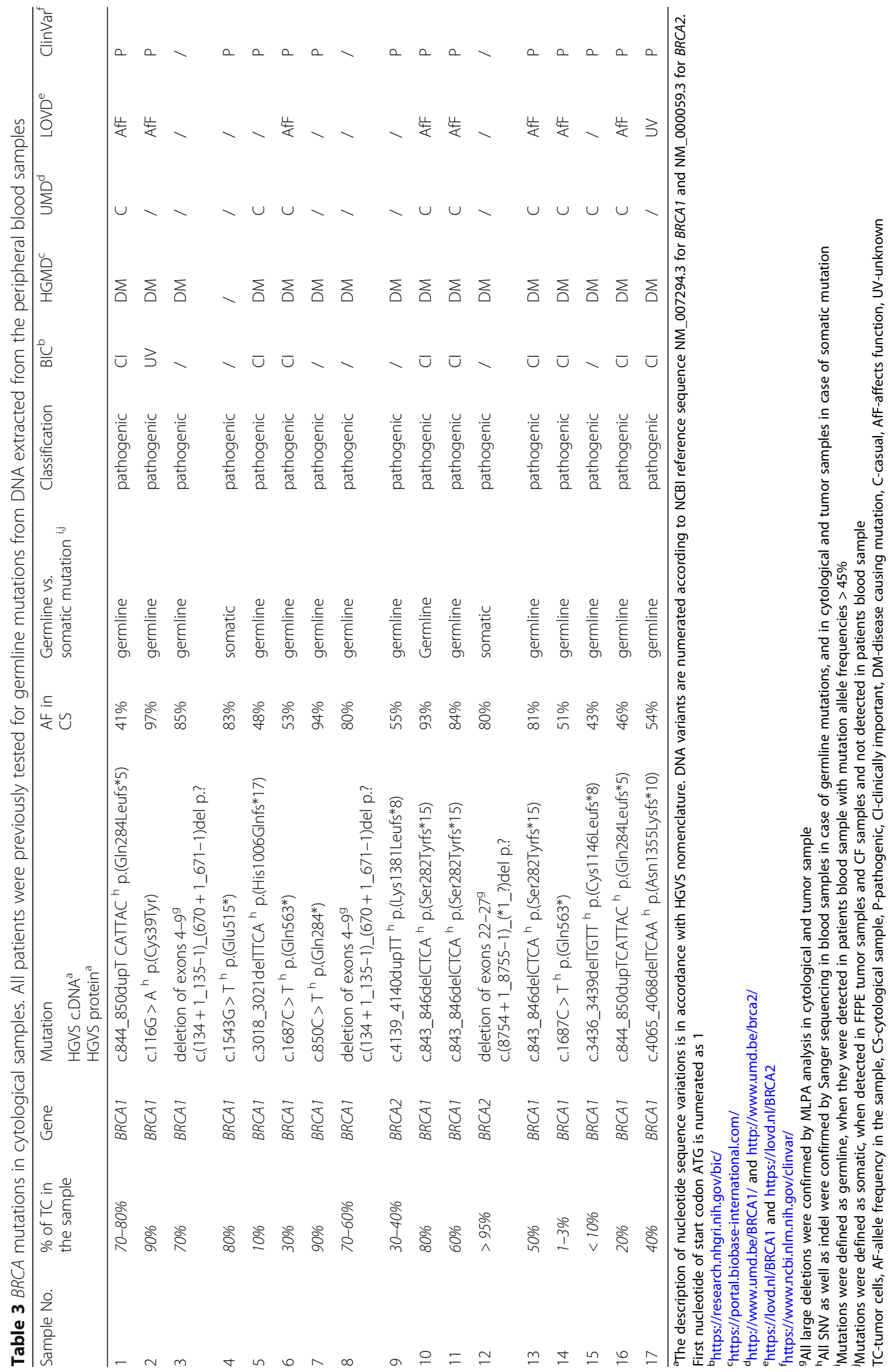


positive family history of breast and ovarian cancer. However, this is somehow in agreement with the results of Alsop et all who also reported that $44 \%$ of $B R C A$ positive patients in Australia presented with no family history of breast and ovarian cancer [7].

Beside a positive family history, multiple primary cancers is another important aspect of hereditary cancer syndromes. In our study we had a complete data for 32 patients and $6(18,7 \%)$ of them had an additional breast cancer diagnosed before HGSC was diagnosed. Family history was positive in $4 / 6(66,7 \%)$ of them. Mean age (at the time of diagnosis of $\mathrm{OC}$ ?) of patients with germline $B R C A 1 / 2$ was 56,9 years (range 43-68). As expected, mean age at the time of diagnosis of $B R C A$ negative patients was higher, 61,7 years (range 40-77).

According to the NCCN guidelines genetic testing should be considered in appropriate high risk individuals where it will impact the medical management of the tested individual and/or their family members at risk. Ideally the probability of mutation detection should be more than $10 \%$ according to the family tree [33]. Since germline mutation rate according to the results of our study and also results of other already mentioned published studies by far exceed $10 \%$, we are facing a similar situation like in patients with medullary thyroid cancer, where genetic counselling and testing is recommended in all patients and not only in those with a positive family history [34]. All patients with epithelial ovarian cancer (including HGSC) should be offered genetic counselling and testing regardless of family history.

Results of our study demonstrated a $100 \%$ concordance of $B R C A 1 / 2$ testing from peripheral venous blood and different cytological samples for detecting a germline mutation in HGSC patients. As such, this approach could simplify and reduce testing rates in OC patients. Patients with negative cytological or histological $B R C A$ test results do not need additional genetic testing for germline BRCA mutations. Only patients who have a positive cytological or histological $B R C A$ test result require additional genetic testing to rule out or confirm germline mutation. Considering before mentioned $B R C A$ mutations frequency in HGSC cancer patients, more than $70 \%$ of HGSC patients would need only cytologicaly derived BRCA mutation test. For 100 cytological or histological samples an additional 30 tests (130 tests altogether) have to be done to differentiate between somatic and germline mutations. On the other hand, if we start with 100 germline mutation tests first and 30 of them will result positive, we need additional 70 tests (170 tests altogether) to differentiate between somatic and germline mutations. Not to mention that by such an approach all patients need to be genetically counselled. Histological tumor sample (usually provided from initial surgery) is therefore only needed for diagnosis of HGSC, whereas cytologic tumor sample offers a good alternative for $B R C A$ testing.

In this regard, evidences presented in this study strongly suggest clinical significance of cytological samples as a valuable tool for widely use in genetic $B R C A$ testing. Simple, rapid and cost-effective methods (at least $70 \%$ reduction in genetic counselling and genetic testing) of molecular testing, which take full advantage of the limited materials available in the clinical setting, such as cytology samples, are urgently required. This is particularly important in cases of frequent recurrences of disease with limited options for surgical treatment and limited availability of histopathologic samples. Cytological material is already routinely used in clinical setting to confirm recurrence/progression of the disease. Regarding to our study results, it could be used for $B R C A$ re-testing after each recurrence/progression of the disease. Since HGSC patients most of the time have multiple recurrences of disease and become chemoresistant, subjecting them to molecular profiling as part of their care during disease progression, enables clinicians to select targeted personalized therapies according to the mutations detected in their tumors before every treatment enrolment. With maximum confidence data, this will hopefully become a part of clinical pathways in the future.

\section{Conclusions}

There is a $100 \%$ concordance between $B R C A 1 / 2$ mutation testing from cytological and histological samples. Moreover, in all patients with a germline $B R C A 1 / 2$ mutation there is also a $100 \%$ concordance between $B R C A 1 / 2 \mathrm{mu}-$ tation testing from cytological and blood samples.

Cytological samples with adequate percentage of tumor cells are equivalent to FFPE samples in determining $B R C A$ mutations and could replace FFPE in BRCA $1 / 2$ gene mutation profiling in HGSC patients. Additional benefits when cytological samples are tested, could be in faster processing of genotyping and earlier informative report for clinician as well as in potentially reduced number of genetic testing.

\footnotetext{
Abbreviations

AF: Allele frequency in the sample; AfF: Affects function; AS: Ascites; C: Casual; Cl: Clinically important; CS: Cytological samples; DM: Disease causing mutation; ECC: Endogenous creatinine clearance; ELN: Enlarged lymph nodes; FFPE: Formalin-fixed paraffin-embedded; HE: Hematoxylin and Eosin;; HG: Homologous recombination; HGSC: High grade serous cancer; Indel: Insertion and deletions; ITH: Intratumoral genetic heterogeneity; NGS: Next Generation Sequencing; OC: Ovarian cancer; P: Pathogenic; PARPi: Poly(ADP-ribose) polymerase inhibitors; PE: Pleural effusion; PFS: Progression-free survival; SNV: Single nucleotide variants; TC: Tumor cells; TSCA: TruSeq Custom Amplicon Kit; TSCancer: TrueSight Cancer panel; UV: Unknown
} 


\section{Funding}

- Republic of Slovenia, Ministry of Health (P3-0289(C)): financial support for publication of the manuscript

- Institute of Oncology Ljubljana (Slovenia): financial support for all procedures needed for design of the study, collection, analysis and in writing the manuscript,

- AstraZeneca (UK): financial support for providing genetic analysis kits.

\section{Availability of data and materials}

The datasets used and/or analyzed during the current study are available from the corresponding author on reasonable request.

\section{Authors' contributions}

Conceptualization: ES; Methodology: ES, SN, ZPM, BG; Collection of samples: AG; Formal analysis and investigation: ZPM, BG, VS, SN; Genetic counselling: MK; Writing - original draft preparation: AG; Writing - reviewing, editing: ES, $\mathrm{MH}, \mathrm{SN}$. All authors read and approved final manuscript.

\section{Ethics approval and consent to participate}

- National Ethics Committee and Institutional Ethics Committee of Institute of Oncology Ljubljana (Ljubljana, Slovenia), 27/07/2015, NMEC 100/05/15.

- Written consent to participate in the study was obtained from each participant.

\section{Consent for publication}

Not Applicable.

\section{Competing interests}

The authors declare that they have no competing interests.

\section{Publisher's Note}

Springer Nature remains neutral with regard to jurisdictional claims in published maps and institutional affiliations.

\section{Author details}

'Department of Gynecologic Oncology, Institute of Oncology Ljubljana, Zaloška cesta 2, 1000 Ljubljana, Slovenia. ${ }^{2}$ Department of Molecular diagnostics, Institute of Oncology Ljubljana, Zaloška cesta 2, 1000 Ljubljana, Slovenia. ${ }^{3}$ Department of Surgical Oncology, Institute of Oncology Ljubljana, Zaloška cesta 2, 1000 Ljubljana, Slovenia. ${ }^{4}$ Department of Cythopathology, Institute of Oncology Ljubljana, Zaloška cesta 2, 1000 Ljubljana, Slovenia. ${ }^{5}$ Department of Pathology, Institute of Oncology Ljubljana, Zaloška cesta 2, 1000 Ljubljana, Slovenia. ${ }^{6}$ Department of Genetic counselling, Institute of Oncology Ljubljana, Zaloška cesta 2, 1000 Ljubljana, Slovenia. ${ }^{7}$ Department of Medical oncology, Institute of Oncology Ljubljana, Zaloška cesta 2, 1000 Ljubljana, Slovenia.

\section{Received: 10 December 2018 Accepted: 27 March 2019} Published online: 02 April 2019

\section{References}

1. Stratton JF. Contribution Of Brca1 Mutations To Ovarian Cancer; 1997. p. 1125-30.

2. Pal T, Permuth-wey J, Betts JA, Krischer JP, Ph D, Fiorica J, et al. BRCA1 and BRCA2 Mutations Account for a Large. 2005.

3. Zhang S, Royer R, Li S, McLaughlin JR, Rosen B, Risch HA, et al. Frequencies of BRCA1 and BRCA2 mutations among 1,342 unselected patients with invasive ovarian cancer. Gynecol Oncol [Internet]. 2011;121(2):353-7 Available from: https://doi.org/10.1016/j.ygyno.2011.01.020.

4. Walsh T, Casadei S, Lee MK, Pennil CC, Nord AS, Thornton AM, et al. Mutations in 12 genes for inherited ovarian, fallopian tube, and peritoneal carcinoma identified by massively parallel sequencing. Proc Natl Acad Sci [Internet. 2011;108(44):18032-7 Available from: https://www.pnas.org/ content/pnas/early/2011/10/13/1115052108.full.pdf.

5. Le Page C, Rahimi K, Köbel M, Tonin PN, Meunier L, Portelance L, et al. Characteristics and outcome of the COEUR Canadian validation cohort for ovarian cancer biomarkers. BMC Cancer. 2018;18(1):1-18.
6. Risch HA, McLaughlin JR, Cole DEC, Rosen B, Bradley L, Kwan E, et al. Prevalence and penetrance of germline BRCA1 and BRCA2 mutations in a population series of 649 women with ovarian Cancer. Am J Hum Genet [Internet]. 2001;68(3):700-10 Available from: https://www.sciencedirect.com/ science/article/pii/S0002929707631097.

7. Alsop K, Fereday S, Meldrum C, DeFazio A, Emmanuel C, George J, et al. BRCA mutation frequency and patterns of treatment response in BRCA mutation-positive women with ovarian cancer: a report from the Australian ovarian cancer study group. J Clin Oncol. 2012;30(21):2654-63.

8. The Cancer Genome Atlas Network. Integrated genomic analyses of ovarian carcinoma. Nature. 2011;474(7353):609-15.

9. Pennington KP, Walsh T, Harrell MI, Lee MK, Christopher C, Rendi MH, et al. Germline and somatic mutations in homologous recombination genes predict platinum response and survival in ovarian. Fallopian Tube, and Peritoneal Carcinomas. 2015;20(3):764-75.

10. Petrillo M, Marchetti C, De Leo R, Musella A, Capoluongo E, Paris I, et al. BRCA mutational status, initial disease presentation, and clinical outcome in high-grade serous advanced ovarian cancer: a multicenter study. Am J Obstet Gynecol [Internet]. 2017;217(3):334.e1-9 Available from: https://doi. org/10.1016/j.ajog.2017.05.036.

11. Weren RDA, Mensenkamp AR, Simons M, Eijkelenboom A, Sie AS, Ouchene $\mathrm{H}$, et al. Novel BRCA1 and BRCA2 tumor test as basis for treatment decisions and referral for. Genetic. 2017;38(2):226-35. https://doi.org/10.1002/humu. 23137. Epub 2016 Nov 9.

12. Ledermann JA, Harter P, Gourley C, Friedlander M, Vergote I, Rustin G, et al. Overall survival in patients with platinum-sensitive recurrent serous ovarian cancer receiving olaparib maintenance monotherapy: an updated analysis from a randomised, placebo-controlled, double-blind, phase 2 trial. Lancet Oncol [Internet]. 2016;17(11):1579-89 Available from: https://doi.org/10. 1016/S1470-2045(16)30376-X

13. Bolton KL, Chenevix-Trench G, Goh C, Sadetzki S, Ramus SJ, Karlan BY, Lambrechts D, Despierre E, Barrowdale D, McGuffog L, Healey S, Easton DF, Sinilnikova O, Benítez J, García MJ, Neuhausen S, Gail MH, Hartge P, Peock S, Frost D, Evans DG, Eeles R, Godw PPE kConFab ICGARN. Association Between BRCA1 and BRCA2 Mutations and Survival in Women with Invasive Epithelial Ovarian Cancer. Jama. 2012;307(4):382-90.

14. Ledermann J, Harter P, Gourley C, Friedlander M, Vergote I, Rustin G, et al. Olaparib maintenance therapy in patients with platinum-sensitive relapsed serous ovarian cancer: a preplanned retrospective analysis of outcomes by BRCA status in a randomised phase 2 trial. Lancet Oncol [Internet]. 2014; 15(8):852-61 Available from: https://doi.org/10.1016/S1470-2045(14)70228-1.

15. Dougherty BA, Lai Z, Hodgson DR, Orr MCM, Sun J, Yelensky R, et al. Biological and clinical evidence for somatic mutations in BRCA1 and BRCA2 as predictive markers for olaparib response in high- grade serous ovarian cancers in the maintenance setting. 2017;8(27):43653-43661.

16. Castellarin M, Milne K, Zeng T, Tse K, Mayo M, Zhao Y, et al. Clonal evolution of high-grade serous ovarian carcinoma from primary to recurrent disease. J Pathol. 2013;229(4):515-24.

17. Norquist B, Wurz KA, Pennil CC, Garcia R, Gross J, Sakai W, et al. Secondary somatic mutations restoring BRCA1/2 predict chemotherapy resistance in hereditary ovarian carcinomas. J Clin Oncol. 2011;29(22):3008-15.

18. Shah RH, Scott SN, MPH BAR, Levine DA, Lin O, et al. Comprehensive mutation profiling by next-generation sequencing of effusion fluids from patients with high-grade serous ovarian carcinoma. Cancer Cythopathol. 2015;123(5):289-97.

19. Bashashati A, Ha G, Tone A, Ding J, Prentice LM, Roth A, et al. Distinct evolutionary trajectories of primary high-grade serous ovarian cancers revealed through spatial mutational profiling. J Pathol. 2013;231(1):21-34.

20. Hoogstraat M, Pagter MS De, Cirkel GA, Roosmalen MJ Van, Harkins T, Duran K, et al. Genomic and transcriptomic plasticity in treatment naive ovarian cancer. 2014;200-211.

21. Alkema NG, Wisman GBA, Van Der Zee AGJ, Van Vugt MATM, De Jong S. Studying platinum sensitivity and resistance in high-grade serous ovarian cancer: different models for different questions. Drug Resist Updat [Internet]. 2016;24:55-69 Available from: https://doi.org/10.1016/j.drup.2015.11.005.

22. Choi YJ, Rhee JK, Hur SY, Kim MS, Lee SH, Chung YJ, et al. Intraindividual genomic heterogeneity of high-grade serous carcinoma of the ovary and clinical utility of ascitic cancer cells for mutation profiling. J Pathol. 2017;241(1):57-66.

23. Ledermann JA. Front-line therapy of advanced ovarian cancer: new approaches. Ann Oncol [Internet]. 2017;28(suppl_8):viii46-50 Available from: https://academic.oup.com/annonc/article/28/suppl_8/viii46/4693818. 
24. Adank, M. A., Brogi E BF et al. Accuracy of BRCA1 and BRCA2 founder mutation analysis in formalin-fixed and paraffin-embedded ( FFPE ) tissue. 2006;337-342

25. Lin J, Kennedy SH, Svarovsky T, Rogers J, Kemnitz JW, Xu A, et al. High-quality genomic DNA extraction from formalin-fixed and paraffin-embedded samples deparaffinized using mineral oil. Anal Biochem [Internet]. 2009;395(2):265-7 Available from: https://doi.org/10.1016/j.ab.2009.08.016.

26. Capoluongo E, Ellison G, López-Guerrero JA, Penault-Llorca F, Ligtenberg MJL, Banerjee S, et al. Guidance statement on BRCA1/2 tumor testing in ovarian Cancer patients. Semin Oncol [Internet. 2017;44(3):187-97 Available from: https://doi.org/10.1053/j.seminoncol.2017.08.004.

27. Plon SE, Eccles DM, Easton D, Foulkes WD, Genuardi M, Greenblatt MS, et al. Sequence variant classification and reporting: recommendations for improving the interpretation of cancer susceptibility genetic test results. Hum Mutat. 2008:29(11):1282-91.

28. Laboratories KD, Genetics M, Health O, Road P, Molecular C, Children N, et al. Standards and guidelines for the interpretation of sequence variants: a joint consensus recommendation of the American College of Medical Genetics and Genomics and the Association for Molecular Pathology. Genet Med. 2015;17(5):405-24.

29. Dattalo P. In: Tony Tripodi PE, editor. Determining sample size. New York: Oxford University Press; 2008. p. 11-55.

30. Hoogstraat M, de Pagter MS, Cirkel GA, van Roosmalen MJ, Harkins TT, Duran $\mathrm{K}$, et al. Genomic and transcriptomic plasticity in treatment-naive ovarian cancer. Genome Res [Internet]. 2014;24(2):200-11 Available from: https://genome.cshlp.org/content/early/2013/11/05/gr.161026.113.

31. Cvelbar M, Hocevar M, Novakovic S, Stegel V, Perhavec A, Krajc M. Genetic counselling, BRCA1/2 status and clinico-pathologic characteristics of patients with ovarian cancer before 50 years of age. Radiol Oncol. 2017; 51(2):187-94.

32. Geisler JP, Hatterman-Zogg MA, Rathe JA, Buller RE. Frequency of BRCA1 dysfunction in ovarian cancer. J Natl Cancer Inst. 2002;94(6):61-7.

33. Clinical N, Guidelines P, Guidelines N. Genetic/Familial High-Risk Assessment : Breast and Ovarian 2018;

34. Wells SA, Asa SL, Dralle H, Elisei R, Evans DB, Gagel RF, et al. Revised American Thyroid Association Guidelines for the Management of Medullary Thyroid Carcinoma. Thyroid [Internet]. 2015;25(6):567-610 Available from: https://www.liebertpub.com/doi/10.1089/thy.2014.0335.

Ready to submit your research? Choose BMC and benefit from:

- fast, convenient online submission

- thorough peer review by experienced researchers in your field

- rapid publication on acceptance

- support for research data, including large and complex data types

- gold Open Access which fosters wider collaboration and increased citations

- maximum visibility for your research: over $100 \mathrm{M}$ website views per year

At $\mathrm{BMC}$, research is always in progress.

Learn more biomedcentral.com/submissions 